\title{
Débat sur une question sociocientifique: expertise de I'information dans le cadre d'une Éducation Citoyenne
}

\section{Catherine Barrué}

Toute réflexion sur la participation des citoyens aux décisions concernant des questions scientifiques controversées doit interroger la scolarisation de celles-ci. Cette étude s'articule autour de l'engagement d'élèves de 11-12 ans dans une conférence de citoyens adaptée au contexte scolaire. Dans une visée d'Éducation Citoyenne critique, il apparaît crucial de s'intéresser à l'expertise documentaire. Quels sont les critères utilisés par les élèves dans l'évaluation des documents et des sources d'informations lorsque les savoirs scientifiques sont controversés? Si les élèves sont dans une stratégie évaluatrice concernant le contenu des documents, peu d'entre eux évaluent les sources. Alors, une attention particulière doit être donnée à l'expertise de l'information pour que des élèves puissent comprendre la complexité des enjeux liés à ces controverses sociales et scientifiques.

\section{La scolarisation d'une question socioscien- tifique pour développer quelles compétences citoyennes?}

Des questions liées aux biotechnologies, à l'environnement, au développement durable, aux choix énergétiques, aux ondes, aux nanotechnologies, agitent régulièrement les espaces social, politique et médiatique. La question de la légitime participation des citoyens aux délibérations et aux prises de décision s'est alors posée dans un cadre démocratique. Cette demande de participation des citoyens a d'ailleurs questionné fortement certains sociologues et historiens des sciences (Barthe, 2006; Bonneuil, 2004; Callon, Lascoumes \& Barthe, 2001). Ils se sont penchés sur les aspirations des citoyens à s'impliquer dans des processus de décision sur des questions techniques et scientifiques. Il est alors, selon eux, impossible de nier la dimension sociale dans des contextes de situations tels que ceux du stockage des déchets nucléaires, des affaires du sang contaminé et de la vache folle, de la culture des OGM. 
La participation des citoyens aux prises de décision sur de telles questions mettant en jeu des savoirs scientifiques en cours de construction renvoie alors à l'éducation des jeunes et, consécutivement, au rôle de l'École. Ces nouvelles questions ont engagé, depuis une douzaine d'années, certains didacticiens des sciences de nombreux pays (Albe, 2007; Grace, 2009; Kolsto, 2001; Hodson, 2003, 2010; Jimenez-Aleixandre \& Pereiro-Muñoz, C, 2002; Sadler, 2004, 2009) à s'interroger sur la manière de former les élèves pour qu'ils puissent se saisir de la complexité de ces questions et des enjeux socio-politiques qui se jouent. La visée éducative est de les outiller de compétences qui leur permettront de participer, dans leur vie d'adulte, aux choix qui se présenteront à eux. La prise en compte de ces questions en classe par l'Institution scolaire, par les enseignants et de leur appropriation par les élèves est alors à examiner.

Ces questionnements scientifiques et techniques qui se mêlent de façon indissociable à des questionnements sociaux (Sadler, 2004), sont reconnus par un courant de recherche comme des questions socioscientifiques (QSSs; socioscientific issues [SSIs] en langue anglaise). En France, ces questions sont tout d'abord entrées dans l'enseignement agricole, puis elles ont été diffusées plus largement dans le contexte scolaire par l'intermédiaire d'une actualité médiatisée et des «éducations à». Elles sont présentes dans des dispositifs d'enseignement pluridisciplinaires au collège comme au lycée et, de façon plus marquée, dans les programmes de Lycée entrés en vigueur en 2011. L'introduction de ces questions dans les curricula interroge les politiques éducatives, puisqu'elles sont présentées en lien avec des enjeux de société et que la visée éducative affichée est l'Éducation Citoyenne [EC]. Cependant, si la visée est explicite dans les termes employés, elle ne l'est ni dans la conception de la citoyenneté qui est envisagée, ni dans ce qui est entendu par Éducation Citoyenne. S'agit-il de former un citoyen capable de s'insérer socialement? S'agit-il de former un citoyen critique engagé dans la construction de la société en participant aux choix qui façonneront son futur? Derrière ces conceptions différentes d'EC, allant d'une EC qualifiée de normative qui viserait à fabriquer un citoyen docile et vertueux à une EC qualifiée d'émancipatrice, en passant par une pluralité de profils de citoyens visés, apparaissent également des visions différentes de ce que doit ou peut être une société démocratique (Barrué \& Albe, 2013). Cela suppose alors de s'interroger sur le citoyen que nous voulons construire et sur l'EC qui serait à privilégier (Audigier, 1999, 2000) pour permettre aux élèves d'appréhender les questions socioscientifiques dans leur vie d'adulte.

Le courant de recherche des QSSs qui défend la scolarisation de telles questions peut alors être interrogé lui aussi quant à la visée éducative qu'il promeut. La plupart des recherches développées par ce courant se revendiquent d'une visée d'EC. Peu d'entre elles explicitent ce qu'elles entendent par éducation citoyenne et quel citoyen elles visent à construire. Les enjeux éducatifs sont cependant clairement explicités: développer la compréhension de la nature de la science, des compétences argumentatives et apprendre à prendre une décision. Enfin, certaines 
études considèrent l'enseignement des QSSs comme un moyen d'apprentissage de connaissances scientifiques ou un moyen de développer des valeurs morales. Derrière ces différents enjeux éducatifs, il est possible d'entrevoir quatre orientations différentes d'EC (Barrué \& Albe, 2013): (1) la formation d'un citoyen engagé dans des actions socio-politiques, (2) la formation d'un citoyen apte à participer aux débats et aux prises de décision ou encore (3) la construction d'un citoyen qui possède des connaissances scientifiques et (4) des valeurs morales en vue de s'insérer socialement.

Si le projet est de promouvoir une citoyenneté souveraine, active et participative, une légitimité du citoyen à participer aux débats et aux prises de décision doit alors être revendiquée. Nous nous positionnons dans une vision émancipatrice d'EC, défendue par une des orientations de la recherche sur les QSSs: celle qui vise à construire un citoyen capable de participer aux discussions et aux prises de décision en matière de questions socioscientifiques. Nous considérons alors, à la suite de certains chercheurs qui s'inscrivent dans cette orientation (Grace, 2009; Hogan, 2002; Jimenez-Aleixandre \& Pereiro-Muñoz, 2002; Kolstø, 2000, 2001), que la mise en œuvre des QSSs en classe est à considérer en relation avec des situations sociales dans lesquelles l'apprentissage de l'argumentation, l'appropriation de connaissances, l'évaluation des discours des experts et de l'information et les prises de décision peuvent être considérés comme des compétences citoyennes. Dans ce cas, les sciences ne peuvent pas être envisagées comme un produit fini prêt à consommer par les élèves mais comme un objet discutable en construction.

Ces compétences sont mises en oeuvre dans des débats en classe pour l'étude de nombreuses QSSs. Elles deviennent alors des objets de recherche développés dans les dispositifs impliquant essentiellement des lycéens. Il peut s'agir d'organiser des débats pour agir sur des instance locales (Hogan, 2002; Jimenez-Aleixandre \& Pereiro-Muñoz, 2002) ou de délibérer pour prendre une décision informée sur le dépistage de maladies héréditaires (Levinson, 2004) ou sur des prises de décision de politiques globales comme la culture des OGM (Böttcher \& Meisert, 2013). Des jeux de rôles sont organisés sur des questions liées aux nanotechnologies (Hingant, 2013). D’autres recherches développent des dispositifs délibératifs tels que des simulations de débats publics ou des délibérations impliquant des scientifiques (Molinatti, 2007; Simonneaux, Ducamp, Albe, Simonneaux \& Hirtzin, 2005). Peu de recherches sont menées avec de jeunes élèves du fait de la complexité des questions abordées. Cependant, quelques études (Byrne, Ideland, Malmberg \& Grace, 2014; Pedretti, 1999) montrent que de jeunes élèves (9 à 12 ans) sont capables de s'engager dans des discussions impliquant des QSSs. C'est pourquoi, nous avons fait le choix de mener cette recherche avec des élèves de 12 ans autour de la question des ondes Wi-Fi. Nous avons examiné comment ces élèves pouvaient s'exercer à une citoyenneté critique et émancipatrice lors d'une conférence de citoyens adaptée pour la forme scolaire. Les objets de cette large recherche ont alors porté sur l'expertise de l'information, les savoirs utilisés dans 
l'argumentation des élèves, les interactions verbales et la manière dont les élèves prennent leur décision. Nous avons cherché également à voir s'ils se saisissent des enjeux scientifiques et sociopolitiques liés à la question traitée.

Dans cet article, c'est l'expertise documentaire qui est questionnée puisqu'elle conditionne les savoirs relatifs à la question des ondes Wi-Fi ici traitée et la construction de l'argumentation dans le débat. Nous voulons ainsi documenter la recherche en didactique des sciences sur les caractéristiques des sources retenues par des élèves et sur leurs critères de choix lorsqu'il s'agit de sélectionner des documents sur des QSSs. Nous nous attachons plus précisément à explorer comment, dans le cadre d'un dispositif citoyen, de jeunes élèves expertisent de l'information issue du web en vue de construire une argumentation qu'ils développeront lors d'un débat. L'expertise de l'information étant, bien entendu, un des objets d'un champ de recherche en science de l'information et de la communication. Cependant, dans notre cadre particulier des QSSs, cette expertise est centrée sur un type particulier d'information: celle relative aux sciences en train de se faire. Cela nous ramène alors dans un champ de recherche qui interroge plus largement l'Éducation Citoyenne en lien avec les QSSs. Ce n'est donc pas l'expertise d'information pour elle-même qui nous intéresse mais ce qu'elle implique dans l'étude d'une QSS à des fins d'Éducation Citoyenne. Mais quelle Éducation Citoyenne est alors à considérer?

\section{Un cadre d'Éducation Citoyenne critique pour L'étude d'une QSS: L'expertise de L'information}

Si la citoyenneté apparait comme un concept mouvant et dynamique et que les enjeux éducatifs de la scolarisation des QSSs sont guidés par la construction d'un citoyen critique qui a un rôle à jouer dans la participation démocratique aux débats de société et aux prises de décisions qui s'y rattachent, il est alors nécessaire d'envisager une Éducation Citoyenne exigeante que nous avons qualifiée d'Éducation Citoyenne critique $[\mathrm{ECc}]$. Cette ECc va bien au delà du développement de compétences sociales visant à coexister pacifiquement et qui sont celles visées de manière prégnante dans les curricula prescrits pour les jeunes élèves (Barrué \& Albe, 2013). Elle doit contribuer à construire les compétences nécessaires pour débattre de façon argumentée et documentée, pour évaluer de l'information, pour comprendre les enjeux économiques, sociaux et politiques afin de peser sur les décisions. Cette visée de l'ECc peut s'appliquer à de nombreuses questions sociétales. Selon nous, elle devient primordiale lorsqu'il s'agit de composer avec des QSSs qui impliquent des connaissances scientifiques non stabilisées. Cela rend d'autant plus complexe la tâche du citoyen lorsqu'il est confronté à des informations contradictoires, à la compréhension de la nature même de la science et aux intérêts qu'elles sous-tendent. Ainsi, notre recherche se place dans l'approche théorique du modèle d' 'Éducation Citoyenne critique pour l'étude 
d'une question socioscientifique» (Barrué, 2014, p. 146). Il prend en compte les conditions de scolarisation d'une QSS dans une visée d'ECc avec une double visée: construire des dispositifs d'enseignement et de recherche et être un outil d'analyse de ceux-ci. La construction de cette modélisation souple s'appuie sur des éléments théoriques et des données empiriques, identifiés à travers des revues de littérature en socio-politique et de recherche en didactique des sciences sur la scolarisation des QSSs. Il prend en compte cinq dimensions qui caractérisent une ECc et certains éléments de la scolarisation des QSSs (Albe, 2007) qui les orientent: (1) la dimension "connaissances», (2) la dimension «argumentation», (3) la dimension «enjeux», (4) la dimension «information» et (5) la dimension «engagement».

C'est plus précisément la dimension «information» de cette modélisation qui constitue le cadre de la recherche développée dans cet article. Elle est adossée à la philosophie de Dewey (2003) qui défend une «citoyenneté active et informée». Elle doit être construite sur la formation d'un public actif mais aussi sur «sa capacité d'enquête». Cette conception se rapporte alors au développement des capacités du citoyen à s'informer. Face à la perte de confiance dans les représentants politiques lors des différentes affaires telles que "la vache folle» et «le sang contaminé», les citoyens se sont tournés vers d'autres sources d'information. Ces nouveaux espaces, qualifiés par Callon, Lascoumes et Barthe (2001) de «forums hybrides», permettent au citoyen de relativiser la valeur des informations scientifiques contradictoires. Ils donneraient une information plus discutée que celle développée lors des simulacres de débats présents dans l'espace télévisuel (Halimi, 1999). La télévision, premier média de masse touchant des citoyens de tous âges et de toutes couches sociales, est souvent accusée de façonner les discours, d'être dépendante de ses financements, de l'audimat (Bourdieu, 1996) et de s'adapter à la demande de son public. Dans ce cas, que deviennent la pluralité des points de vue et la transparence des informations? Le recours à des sources d'information comme la TV et Internet, deuxième moyen d'information des Français, pose la question du décryptage de ces médias et de la formation du citoyen à cet exercice: qui parle? Au nom de qui? Dans quel but? Qui donne les infos à qui? Qui finance ces médias?

La question d'une formation à l'expertise de l'information est prise en compte par certains didacticiens dans l'étude d'une QSS. Kolstø (2001) étudie les stratégies des élèves de 15-16 ans lorsqu'ils choisissent des documents "papier» et les critères suivant lesquels ils le font. Witzig, Halverson, Siegel et Freyermuth (2011) s'intéressent quant à eux à l'évaluation des sources Internet et des informations recueillies qui contribuent à construire les connaissances et les opinions d'élèves Ingénieurs en Agronomie sur la question de la recherche sur les cellules souches. L'enjeu éducatif de ces chercheurs est de former les élèves à être des évaluateurs critiques des sources qui délivrent l'information. Les auteurs examinent la manière dont ceux-ci évaluent ces sources et ce qu'ils disent de l'influence de cette évaluation sur leurs opinions et sur leur compré- 
hension de la question. Ces étudiants ont aussi pris en considération la facilité de compréhension des sites visités, les détails donnés, la langue, la facilité de navigation et le statut des auteurs des articles en fonction de leur domaine. Ils ont sélectionné des sites sur la question des cellules souches qui sont essentiellement des sites de revues scientifiques, des sites médicaux, des sites généralistes, des sites universitaires et scolaires et des sites gouvernementaux. Quelles que soient leurs opinions sur les cellules souches, ils ont considéré que les sources retenues étaient globalement crédibles. Pour ce qui est de l'objectivité des sites, ils rapportent que les sites médicaux, les sites scolaires, universitaires et les sites gouvernementaux sont objectifs car ils contiennent des informations factuelles et peu d'opinions. Cependant certains étudiants ont mis en cause les sites gouvernementaux: ils délivreraient de l'information biaisée du fait qu'ils rapportent uniquement l'orientation du gouvernement. Par contre, les sites de journaux scientifiques sont considérés comme objectifs par la moitié des étudiants. La plupart de ces étudiants rapportent que les informations nouvelles apprises grâce à cette évaluation critique des sources ont renforcé leur compréhension de la science fondamentale relative aux cellules souches.

Comme dans les recherches précédentes, l'enjeu éducatif de notre recherche est de former les élèves à l'expertise des sources et de l'information suivant les deux critères pris en compte dans la dimension «information» de notre modélisation: rechercher et expertiser de l'information. Ainsi, il n'est pas possible de faire l'économie, dans une visée d'ECc, d'une formation à la recherche et à l'expertise d'informations, spécialement lorsqu'il s'agit de QSSs qui mettent en jeu des informations contradictoires et souvent provisoires du fait des liens qu'elles entretiennent avec des savoirs non établis. La formation des élèves à l'expertise de l'information s'impose comme une nécessité et ce dès le plus jeune âge, si le but recherché est de contribuer à la construction d'un citoyen capable de démêler le «vrai» du «faux» et d'appréhender les enjeux qui orientent l'information pour tenter d'échapper à une forme de manipulation.

Il apparaît donc important de déterminer sur quels critères les élèves accordent du crédit ou non aux documents retenus et à leurs sources pour ensuite déterminer le poids qu'ils ont dans la construction des savoirs (Barrué, 2014) utilisés dans l'argumentation lorsque ces élèves débattent de la question des ondes $\mathrm{Wi}$-Fi. Quelles sont les raisons qui poussent les élèves à retenir comme valides certaines informations? Quels sont les indicateurs de choix d'une source d'information? À quels savoirs se rapportent-ils? 


\section{Méthodologie de l'étude}

\section{Les participants}

Vingt-neuf élèves âgés de 12 ans (14 filles et 15 garçons) de la même classe de cinquième d'un collège ont été engagés dans un processus participatif organisé comme une Conférence de Citoyens, adaptée pour le contexte scolaire. Trois enseignants de la classe (un professeur de Sciences Physiques, un professeur de Sciences de la Vie et de la Terre et un professeur d'histoire-géographie) ont aidé la chercheure. Ce collège de Région Bretagne ne présente aucune particularité.

\section{La question des ondes électro-magnétiques}

Les 29 élèves de cette étude ont dû s'informer sur la question des ondes électromagnétiques Wi-Fi en vue de se préparer pour un débat. Les effets des ondes électro-magnétiques utilisées par la téléphonie mobile et le $\mathrm{Wi}$-Fi sont actuellement toujours controversés dans la sphère scientifique comme dans la sphère sociale, comme peuvent le montrer des cartographies de cette controverse ${ }^{1}$. Les savoirs scientifiques ne sont toujours pas stabilisés et mettent en évidence des résultats incertains, voire contradictoires. Ce qui est certain, c'est que les incertitudes demeurent et que des scientifiques, comme des autorités sanitaires et des associations de citoyens font des recommandations de prudence concernant l'exposition à ces ondes. Les scientifiques sont unanimes sur le fait qu'une exposition aux champs magnétiques génère des effets thermiques. Par contre, l'impact des effets thermiques sur la santé ne fait pas consensus. Certains considèrent qu'il n'y a pas de danger; d'autres signalent une altération possible de l'ADN, une détérioration éventuelle des fonctions auditives et cognitives. Certaines études, notamment pratiquées sur des animaux, ont établi que les champs électromagnétiques rendraient perméables la barrière hémato-encéphalique qui protège le cerveau et que cela engendrerait des migraines et l'apparition de tumeurs cancéreuses. D'autres études ont abouti à des résultats contradictoires. Il est difficile de comparer ces résultats car les méthodes de recherche different. Les choix de certaines méthodologies et pratiques de recherche sont d'ailleurs remis en cause au sein même de la communauté scientifique. Des études sont menées dans de nombreux pays à l'initiative de différents groupes, comme des associations citoyennes, des laboratoires d'Hôpitaux public, de l'OMS, des fournisseurs d'accès à internet, qui ne sont pas porteurs des mêmes intérêts. L'état des recherches met en lumière que les résultats sont incertains, que les savoirs scientifiques sont controversés et porteurs d'intérêts, bien que l'enjeu majeur soit de réduire les incertitudes pour le citoyen.

\section{Le dispositif de conférence de citoyens construit pour le contexte scolaire}

Ce protocole de débat s'arc-boute sur la procédure participative du modèle des conférences de citoyens, mais modifiée pour s'adapter au cadre ordinaire 
d'une classe de collège. Dans une conférence de citoyens, les membres du panel n'ont aucune connaissance spécifique sur la question traitée. Ils formulent des questions pour le débat et participent à la sélection des experts qui y répondront. Après le débat, ils donnent leur avis à propos de ce qu'il serait souhaitable de faire. Comme les citoyens du panel, les élèves de cette étude ont à faire des recommandations au directeur du collège; ils doivent choisir le système Wi-Fi ou Ethernet pour l'installation d'Internet dans leur collège. Pour cela, 11 séances de deux heures chacune ont été mises en place sur une durée d'environ 3 mois.

Première étape: c'est une première séance (séance 1) au cours de laquelle l'activité, les modalités de travail ainsi que la question à traiter ont été présentées aux élèves. C'est au cours de cette séance que les élèves ont identifié les domaines d'expertise qu'ils souhaitaient étudier pour traiter la question d'actualité du collège: «Nous voulons installer Internet partout dans le collège. Devons-nous installer du Wi-Fi ou du filaire?». Les élèves ont identifié trois domaines d'expertise sur lesquels ils voulaient s'informer: (1) les ondes Wi-Fi et la santé, (2) les performances techniques des deux systèmes possibles et (3) l'aspect économique.

Deuxième étape: elle est l'équivalent de la formation du panel lors d'une conférence de citoyens. Comme il n'était pas possible de faire venir différents experts dans la classe, la formation des élèves s'est faite par l'étude de documents choisis et extraits du web par eux-mêmes. Le choix de la recherche libre de documents est en accord avec notre posture d'EC critique qui met en évidence la nécessité pour tout citoyen ordinaire de s'informer en vue d'une prise de décision informée et documentée. Les élèves ont travaillé par groupes de 3 ou 4. Les 9 groupes ont sélectionné trois documents lors d'une séance (séance 2) pour répondre aux questions qu'ils se posaient sur les ondes Wi-Fi et la santé. Ensuite, chacun des 9 groupes a extrait 3 à 4 arguments de cette documentation lors de la séance suivante (séance 3). Ensuite, une séance en classe entière a été menée, au cours de laquelle tous les arguments rassemblés dans un tableau par la chercheure ont été mis en commun afin de partager les informations, les éclaircir et produire une première synthèse sur les aspects «santé» de la question. Cette procédure a été renouvelée pour les deux autres domaines d'expertise soient les "performances techniques» des systèmes (séances 5, 6 et 7) et les "aspects économiques» (séances 8,9 et 10 ).

Pour chaque document sélectionné lors des séances de recherche documentaire, les élèves ont eu à compléter une feuille «Renseignements sur le document». Ce document devait comporter le titre du document choisi, l'adresse internet de la source, des renseignements sur les auteurs (Qui sont-ils? Pour qui travaillent-ils? Pour qui écrivent-ils?) et la réponse à la question suivante: «Pourquoi avez-vous choisi ce document?». Nous cherchions, par l'utilisation de cet outil, à aider les élèves à s'engager dans la voie de l'expertise en leur donnant des pistes pour s'interroger quant à la validité des informations recueillies. D'autre part, cet outil a permis également de fournir des données pour la recherche. 
Troisième étape: le débat s'est tenu lors de la séance 11 durant deux heures. La première heure, chaque élève a relu les trois rapports de synthèse pour se remémorer les informations. Après cela, ils ont débattu environ pendant une heure. La dernière demi-heure de la séance a été consacrée à la prise de décision et à la rédaction des premières recommandations. La chercheure a été la modératrice du débat et a adopté une position d'impartialité engagée (Kelly, 1986). Les trois enseignants étaient en posture d'observateurs actifs.

\section{Les données récoltées et l'outil d'analyse de L'expertise de l'information}

Notre corpus d'analyse est constitué des 58 feuilles «Renseignements sur le document" complétées par les élèves pour chaque document sélectionné dans l'Internet. Ce sont spécifiquement les réponses à la question «Pourquoi avez-vous choisi ce document?» qui ont été analysées.

L'analyse a été menée à l'aide d'un outil construit à partir des travaux de Kolstø (2001) et Klosterman et Sadler (2008). Certains autres éléments que nous avons jugés pertinents au regard d'une première lecture des réponses des élèves et non pris en compte dans les recherches de Kolstø (2001) et Klosterman et Sadler (2008) ont été ajoutés. En 2001, Kolstø a caractérisé les différentes stratégies des élèves lorsqu'ils jugent les informations qui sont impliquées dans leur prise de décision à propos du lien éventuel entre la leucémie infantile et les lignes à hautes tensions. L'analyse qualitative des verbatims des entretiens d'élèves conduit le chercheur à mettre en lumière des catégories émergentes de stratégies d'élèves quant à la prise en compte des informations liées à la question traitée: certains élèves se centrent sur le contenu de l'information (content-focused) tandis que d'autres se centrent sur l'autorité de la source associée à l'information (sources of knowledge and information), c'est-à-dire les auteurs associés à ces informations. Parallèlement, le chercheur identifie deux stratégies de la part des élèves: une posture d'acceptation ou une posture d'évaluation du contenu et/ou des sources des informations. Dans notre recherche, les informations sont exclusivement des ressources issues de sites web. C'est pourquoi, nous nous sommes aussi référée aux travaux de Klosterman et Sadler (2008) qui portent sur l'évaluation des web-ressources concernant les QSSs. Ils ont construit un outil d'évaluation de cette forme d'information en se focalisant sur quatre critères: (1) l'actualité du sujet, (2) le contenu, (3) l'autorité et (4) la facilité d'utilisation du site. Le test de la grille d'analyse, construite à partir de ces critères sur un petit échantillon des feuilles «informations sur le document», nous a conduite à faire des apports à propos de l'évaluation et de l'acceptation du support, soit le site web, source de l'information. Chaque feuille "Information sur le document» a été analysée à l'aide de la grille présentée ci-après (tableau 1). Une analyse quantitative a aussi été menée. Chaque réponse à la question «Pourquoi avez vous choisi ce document?» a été classée dans une ou plusieurs catégories en fonction des indicateurs de la grille. Elles ont été comptabilisées par catégorie et par domaine d'expertise pour fournir des résultats quant aux critères de sélection des documents. 


\section{Tableau 1. Grille d'analyse pour l'évaluation et l'expertise des informations retenues par les élèves}

\begin{tabular}{|c|c|c|}
\hline $\begin{array}{l}\text { Attitude } \\
\text { centrée sur }\end{array}$ & $\begin{array}{c}\text { Acceptation } \\
\text { (pas de remise en } \\
\text { cause) }\end{array}$ & $\begin{array}{c}\text { Évaluation } \\
\text { (remise en cause ou discussion) }\end{array}$ \\
\hline $\begin{array}{l}\text { LE CONTENU } \\
\text { (du document issu } \\
\text { du web-site) }\end{array}$ & $\begin{array}{l}\quad \begin{array}{l}\text { Accepte les } \\
\text { connaissances }\end{array} \\
\text { Aucune remise en } \\
\text { cause des informa- } \\
\text { tions }\end{array}$ & $\begin{array}{l}\text { Évalue les connaissances } \\
\text { Les indicateurs } \\
\text { - Évaluation des connaissances } \\
\text { «Accord»: les chercheurs sont d'accord entre eux et la } \\
\text { question est dans leur domaine d'expertise. } \\
\text {-«Forme»: chiffres, relations causales; détails et propos } \\
\text { clairs. } \\
\text { - «Pertinence»: adéquation avec les objectifs des élèves } \\
\text { «Concordance»: avec les connaissances des élèves; si les } \\
\text { informations sont non contradictoires. } \\
\text {-«Qualité de la recherche»: méthodes, preuves, si les } \\
\text { résultats semblent justes; précision de l'info, objectivité; } \\
\text { info récente, importante et unique; profondeur du } \\
\text { propos; s'appuie sur de la bibliographie. } \\
\text { - «Évaluation autonome»: les élèves se centrent sur } \\
\text { l'importance d'entendre tous les acteurs impliqués avant } \\
\text { de prendre une décision. } \\
\text { Ils sont conscients de la nécessité d'avoir leur propre } \\
\text { évaluation. }\end{array}$ \\
\hline $\begin{array}{l}\text { L'AUTORITÉ } \\
\text { (auteur du } \\
\text { document et } \\
\text { origine du site) }\end{array}$ & $\begin{array}{l}\text { Accepte l'autorité } \\
\text { Les indicateurs: } \\
\text { - Confiance dans } \\
\text { les chercheurs } \\
\text { - Confiance dans } \\
\text { un acteur spéci- } \\
\text { fique; prestige }\end{array}$ & $\begin{array}{l}\text { Les indicateurs: } \\
\text { - Analuse l'autopinion /risque: les acteurs prennent en consi- } \\
\text { dération les risques liés à la question; ils sont d'accord } \\
\text { avec l'opinion des élèves. } \\
\text { - Analyse des intérêts. } \\
\text { - Neutralité. } \\
\text { - Compétence: ils ont des connaissances sur le sujet; } \\
\text { adéquation entre le sujet traité et la fonction. } \\
\text { - Origine du site: Si anonyme, appel à la responsabilité. }\end{array}$ \\
\hline $\begin{array}{l}\text { LE SUPPORT } \\
\text { (Usability) }\end{array}$ & $\begin{array}{l}\text { Accepte le support } \\
\text { Indicateur: } \\
\text { - Attractivité }\end{array}$ & $\begin{array}{l}\text { Envalue le support } \\
\text { Indicateurs } \\
\text { - Utilisation aisée. } \\
\text { - Compréhension facile (vocabulaire adapté). } \\
\text { - Navigation facile. } \\
\text { - Adéquation du site avec le problème traité (type de } \\
\text { site). }\end{array}$ \\
\hline
\end{tabular}




\section{Les résultats}

Macro analyse des stratégies d'évaluation ou d'acceptation des documents

Le nombre de réponses à la question "Pourquoi avez-vous choisi ce document?» est similaire pour les trois domaines d'expertise retenus, soit «la santé», "les performances techniques» et «l'économie». Ces résultats sont présentés dans le tableau ci-après (Tableau 2).

Tableau 2. Analyse quantitative des réponses des élèves à "Pourquoi avez-vous choisi ce document?"

\begin{tabular}{lllll} 
Domaine & Santé & $\begin{array}{l}\text { Performances } \\
\text { Techniques }\end{array}$ & Économie & Total \\
\hline Nombre de réponses & 20 & 18 & 20 & 58 \\
\hline Évaluation & 16 & 17 & 17 & 50 \\
\hline -Évaluation du contenu & 10 & 15 & 15 & 40 \\
-Evvaluation de l'autorité & 6 & 1 & 0 & 7 \\
-Évaluation du support & 0 & 1 & 2 & 3 \\
\hline Acceptation & 4 & 1 & 3 & 8 \\
\hline -Acceptation du contenu & 1 & 0 & 1 & 2 \\
-Acceptation de l'autorité & 3 & 1 & 0 & 4 \\
-Acceptation du support & 0 & 0 & 2 & 2
\end{tabular}

Les 50 feuilles réponses "Renseignements sur le document" sont classées selon des critères d'évaluation contre 8 classées selon des critères d'acceptation. Les élèves ont été majoritairement dans une attitude d'évaluation du contenu, de l'autorité et du support des documents. Cette évaluation s'est faite dans des proportions similaires, quels que soient les domaines d'expertise.

Lorsque les élèves justifient le choix d'un document retenu dans l'Internet, 42 réponses sur 58 sont centrées sur le contenu du document, comme critère de choix. Parmi ces 42 réponses, 40 d'entres elles concernent une évaluation de celui-ci alors que 2 montrent une stratégie d'acceptation du contenu. Lorsqu'ils évaluent le contenu, ils le font de manière plus significative dans les domaines des performances techniques et de l'économie: 10 réponses sur 20 pour le domaine santé, soit 50\%; 15 réponses sur 18 pour le domaine des performances techniques, soit $83 \%$; 15 réponses sur 20 pour le domaine de l'économie, soit $75 \%$.

Puis 11 réponses sur 58 sont centrées sur l'autorité associée au document, principalement pour les documents relatifs au domaine de la santé: 7 réponses sont évaluatrices de l'autorité liée au document et 4 montrent une attitude d'acceptation de la source sans aucune discussion.

Enfin, 5 réponses sur 58 concernent le support documentaire (la page ou le site consulté). Cette fois, c'est le domaine des performances techniques qui 
est représenté avec 3 réponses évaluatrices et 2 montrant une acceptation du document par acceptation du support.

Bien que le taux de réponses soit faible, il semble que ces élèves ont globalement choisi les documents par leur évaluation quel que soit le domaine d'expertise. Ils se centrent principalement sur le contenu de celui-ci même si des différences peuvent être rapportées. Concernant le domaine de la santé, l'évaluation s'est faite en référence à l'autorité associée au document. Pour les deux autres domaines, soit les domaines des performances techniques et de l'économie, c'est significativement l'évaluation du contenu qui a guidé les choix.

\section{Micro-analyse de La stratégie d'acceptation \\ Acceptation du contenu}

Certains groupes ont choisi le document parce qu'ils «n'en trouvaient pas d'autres». Nous pouvons donc considérer qu'ils l'ont sélectionné par défaut et non pas en analysant réellement son contenu.

\section{Acceptation de l'autorité}

Seuls huit groupes font état d'une acceptation du document sans évaluation préalable. Quatre d'entre eux font état d'un choix lié à la confiance qu'ils ont dans l'autorité associée. Cela concerne le domaine de la santé. Les réponses ci-dessous de ces groupes mettent en lumière une confiance dans des groupes qu'ils reconnaissent comme des experts: les chercheurs, les ingénieurs et les journalistes spécialisés.

\section{Groupe 7: Parce que c'est des chercheurs et ils savent!}

Groupe 1: On a choisi celui-là parce que c'est des ingénieurs qui écrivent.

Groupe 7: Ça à l'air d'un site de confiance parce que c'est des journalistes.

L'autorité des scientifiques n'est pas remise en cause et a pour effet de renforcer la validité de l'information.

\section{Acceptation du support du document}

Même si ce ne sont que des réponses minoritaires, l'attractivité du site source semble être est un élément déclencheur dans le choix d'un document hébergé dans ce site.

Groupe 5: Parce que le site était accueillant! Y avait un oeil qui s'ouvre pour entrer!

Groupe 7: Parce que le site nous donnait envie d'ouvrir le document.

Micro-analyse des stratégies d'évaluation

Évaluation du contenu

Les cinq indicateurs explicités dans notre outil analytique pour traiter les réponses des élèves à propos de l'évaluation du contenu et, plus précisément, les connaissances sont: (1) la concordance des discours entre les chercheurs sur une question 
dans leur domaine d'expertise, (2) la forme du document, (3) sa pertinence ou adéquation avec l'objet à traiter, (4) la compatibilité des informations entre elles et (5) la qualité de la recherche. Le tableau 3 ci-dessous présente la répartition des réponses par indicateur et par domaine.

Tableau 3. Analyse quantitative des réponses des élèves en fonction des indicateurs d'évaluation de contenu.

\begin{tabular}{llllll}
\hline Indicateurs & $\begin{array}{l}\text { La concor- } \\
\text { dance des } \\
\text { discours entre } \\
\text { les chercheurs }\end{array}$ & La forme & La pertinence & $\begin{array}{l}\text { La compa- } \\
\text { tibilité des } \\
\text { informations }\end{array}$ & $\begin{array}{l}\text { La qualité de } \\
\text { la recherche }\end{array}$ \\
\hline Santé & 0 & 4 & 8 & 0 & 3 \\
\hline $\begin{array}{l}\text { Performances } \\
\text { techniques }\end{array}$ & 0 & 3 & 4 & 2 & 8 \\
\hline Économie & 0 & 2 & 5 & 2 & 6 \\
\hline Total & 0 & 9 & 17 & 4 & 17 \\
\hline
\end{tabular}

Les deux indicateurs de choix du document les plus fréquemment identifiés sont la «pertinence» de l'information par rapport à l'objet à traiter et la «qualité de la recherche». La pertinence des informations est l'indicateur qui prédomine pour le domaine de la santé, alors que la qualité de la recherche est prise en compte de façon majoritaire dans le choix des documents relatifs au domaine des performances techniques.

Concernant la pertinence du document, les élèves ont répondu massivement que le contenu du document doit être en adéquation avec la question à traiter. Ils se montrent capables de sélectionner de façon pertinente des documents répondant à leurs besoins. Ils montrent qu'ils se saisissent de l'objet à traiter, comme l'illustrent les exemples qui suivent.

Groupe 9: Parce qu'il répondait aux critères que nous avions choisis, c'est-à-dire le Wi-Fi et la santé.

Groupe 8: Car le document correspondait à notre recherche.

Groupe 5: Parce qu'il contenait tout ce qu'on avait besoin de savoir.

Pour ce qui est de la qualité de la recherche, les élèves justifient leur choix par rapport aux méthodes employées, aux preuves avancées, à la vraisemblance des résultats, à l'importance des informations et à la profondeur des propos tenus.

Groupe 8: Car il démontre l'inefficacité du sans fil. Il prouve que le filaire reste la meilleure manière d'installer Internet chez soi.

Elle est également évaluée par rapport à la précision de l'information, aux justifications apportées et aux efforts d'explication développant des propos clairs.

Un autre aspect pris en compte par les élèves pour attester de la qualité, se rapporte à la quantité importante d'informations apportées par le document et la profondeur du propos développé. 
Groupe 1: Ce document nous propose beaucoup d'arguments.

Groupe 8: Car nous cherchions un document approfondi scientifique.

Groupe 6: Parce que c'était le plus complexe!

Les méthodes développées dans la présentation des informations, les apports conjoints d'informations sur les avantages et les inconvénients semblent avoir aussi guidé leur choix.

La forme a été prise en compte de façon peu significative. Quelques réponses font apparaître des choix liés plus précisément à la forme du document comme la présence de détails et une compréhension aisée: "On a choisi ce document comme il explique bien» et "car ils détaillent bien et il est compréhensible».

La compatibilité des informations entre elles a été très peu prise en compte dans l'évaluation du contenu d'un document par rapport à un autre précédemment lu. Les élèves se montrent peu soucieux de celle-ci. Aucun groupe ne s'est attardé sur la concordance des discours des chercheurs dans un même champ de compétence. Le choix s'est fait pour certains groupes en fonction de la compatibilité des informations avec le choix du système d'installation d'Internet qu'ils défendent.

Groupe 3: Parce qu'il explique que le filaire est moins cher que le Wi-Fi, comme nous.

Groupe 1: Parce qu'il donnait de bons et vrais arguments, que le Wi-Fi est pas efficace!

Il est important ici de nous attarder sur une autre forme d'évaluation du contenu et des connaissances: l'évaluation autonome. Pour Kolstø (2001), l'évaluation autonome se manifeste lorsque les élèves se centrent sur l'importance d'entendre les différents points de vue des différents acteurs concernés par la controverse avant de prendre une décision. Ici, nous considérerons qu'ils évaluent de façon autonome lorsqu'ils choisissent des documents qui développent des informations relatives à différents points de vue dans un même texte informatif. Nous considérons que ces élèves sont alors conscients de la nécessité d'avoir leur propre évaluation à partir d'éléments contradictoires. Neuf réponses sur les 50 explicitent le choix d'un document parce qu'il présente à la fois les avantages et les inconvénients des deux systèmes d'installation d'Internet. Et 6 réponses sur les 50 se réferent à la comparaison du coût des différents types d'installations d'Internet. Par ailleurs, aucun document relatif au domaine de la santé n'a été choisi pour cette raison.

\section{Évaluation de l'autorité}

Nous avons précédemment noté que c'est dans le domaine de la santé que les choix des documents ont été faits par évaluation de l'autorité, même si celui-ci reste minoritaire. Les acteurs cités par les élèves sont les scientifiques, les associations, les journalistes et des personnes anonymes écrivant dans le site source. Nous avons cinq indicateurs d'analyse explicités précédemment dans notre outil 
d'analyse: (1) l'analyse de l'opinion par rapport aux risques, c'est-à-dire la prise en considération des risques par les différents acteurs reconnus comme autorité par les élèves, (2) l'analyse des intérêts de ceux-ci, (3) leur neutralité, (4) leur compétence dans le domaine traité et (5) l'origine du site faisant office d'autorité. Le tableau 4 ci-après présente la répartition du peu de réponses obtenues par indicateur et par domaine. Nous rappelons que la macro-analyse a montré que peu d'élèves étaient dans une attitude d'évaluation de l'autorité associée au document. Cependant, il nous paraît nécessaire de prendre en compte ces réponses pour dégager un éventuel critère majoritaire qui pourrait être mis en évidence également dans de futures réitérations de ce design.

Tableau 4. Analyse quantitative des réponses des élèves en fonction des indicateurs d'évaluation de l'autorité.

\begin{tabular}{llllll}
\hline Indicateurs & $\begin{array}{l}\text { Analyse des } \\
\text { opinions des } \\
\text { acteurs par } \\
\text { rapport aux } \\
\text { risques }\end{array}$ & $\begin{array}{l}\text { Intérêts des } \\
\text { acteurs }\end{array}$ & $\begin{array}{l}\text { Neutralité des } \\
\text { acteurs }\end{array}$ & $\begin{array}{l}\text { Compétence } \\
\text { des acteurs }\end{array}$ & $\begin{array}{l}\text { L'origine du } \\
\text { site faisant } \\
\text { autorité }\end{array}$ \\
\hline Santé & 3 & 0 & 2 & 3 & 1 \\
\hline $\begin{array}{l}\text { Performances } \\
\text { techniques }\end{array}$ & 0 & 0 & 0 & 0 & 1 \\
\hline Économie & 0 & 0 & 0 & 0 & 0 \\
\hline Total & 3 & 0 & 2 & 3 & 2 \\
\hline
\end{tabular}

Dans les quelques réponses des groupes, quatre indicateurs d'évaluation de l'autorité parmi les cinq ont pu être identifiés. Les élèves n'ont pas abordé la question des intérêts des acteurs associés aux documents sélectionnés, mais uniquement leurs rapports aux risques potentiels, leur neutralité et leurs compétences. Deux groupes d'élèves ont discuté la question de l'origine du site web reconnu comme autorité. Cette évaluation de l'autorité ne concerne que des documents relatifs au domaine de la santé. Un seul groupe a eu recours à l'évaluation de l'autorité pour le domaine des performances techniques.

Dans la réponse ci-dessous donnée par le groupe 3, la justification du choix du document montre que ces élèves attribuent aux scientifiques une mission de protection de la population.

Groupe 3: Car les scientifiques expliquent les effets du Wi-Fi, les maladies que l'on peut avoir et comment se protéger et comment limiter son exposition.

Ils ont choisi ce document parce que les scientifiques expliquent les effets du Wi-Fi mais donnent aussi des indications pour lutter contre les risques.

Le groupe 4 justifie son choix par "parce que c'est des scientifiques qui écrivent sur un site d'association. Parce qu'ils font des recherches sur cela, c'est leur métier». Les scientifiques et les associations sont mis en lien; les élèves attribuent une 
absence d'intérêt aux scientifiques qui écrivent pour une association à qui ils attribuent une forme de neutralité. Dans ce cas, les écrits des chercheurs pourraient légitimer les informations diffusées par des associations de défense de la population.

Ce même groupe d'élève a pris en compte la compétence de ces acteurs c'està-dire l'adéquation entre la question traitée et leur fonction: "Parce que c'est des journalistes et qu'ils travaillent sur la santé».

Ils ont retenu certains documents car les auteurs sont des journalistes écrivant dans des revues liées à la santé. Ces élèves reconnaissent ceux-ci comme compétents car écrivant dans une presse spécialisée. Les élèves questionnent la neutralité des journalistes à travers des connaissances qu'ils ont sur le monde du journalisme, comme l'illustre la déclaration suivante du groupe 10: «Parce que la source d'information vient du Figaro (journal) donc l'information est plus sûre que si on l'avait trouvée sur un blog. L'information aurait été moins sûre car le journal est obligé de vérifier toutes ses informations."

Pour eux, les journalistes se doivent de vérifier leurs informations avant de les diffuser. Ils ne rapporteraient que des faits avérés. A contrario, ils pensent que des faits issus de blog n'ont pas de légitimité si on ne se soucie pas de qui écrit.

\section{Évaluation du site support}

Pour identifier si les élèves évaluent le site support du document, nous nous référons aux quatre indicateurs explicités dans notre outil d'analyse: (1) la facilité d'utilisation, (2) la facilité de compréhension des données fournies, (3) la navigation aisée et (4) le type de site, c'est-à-dire son adéquation à la question traitée. Les trois réponses données par les groupes d'élèves ont été classées suivant ces indicateurs et répertoriées dans le tableau ci-dessous (tableau 5). Nous rappelons ici que la macro-analyse a mis en évidence que peu de groupes avaient une d'attitude évaluatrice du support. Malgré cela, nous notons que les trois réponses sont relatives à un seul indicateur.

Tableau 5. Analyse quantitative des réponses des élèves en fonction des indicateurs d'évaluation du support.

\begin{tabular}{lllll}
\hline Indicateurs & $\begin{array}{l}\text { Facilité d'utili- } \\
\text { sation }\end{array}$ & $\begin{array}{l}\text { Facilité de } \\
\text { compréhension }\end{array}$ & $\begin{array}{l}\text { Facilité de } \\
\text { naviguer }\end{array}$ & $\begin{array}{l}\text { Type de site: } \\
\text { adéquation avec } \\
\text { la question }\end{array}$ \\
\hline Santé & 0 & 0 & 0 & 0 \\
\hline $\begin{array}{l}\text { Performances } \\
\text { techniques }\end{array}$ & 0 & 0 & 0 & 1 \\
\hline Économie & 0 & 0 & 0 & 2 \\
\hline Total & 0 & 0 & 0 & 3 \\
\hline
\end{tabular}


Nous avons identifié uniquement l'indicateur d'évaluation concernant le type de site et son adéquation avec la question traitée. Une seule des trois réponses concerne le domaine des performances techniques et les deux autres le domaine de l'économie. Ces groupes ont choisi le document par évaluation du site source. Leurs réponses sont évaluatrices du site hébergeur et non pas du document en lui-même ou de son auteur. Un des groupes a choisi le document car le site s'adressait à des entrepreneurs au moment où ils étaient en recherche d'informations sur le coût des installations. Ils ont adapté leur recherche à leurs préoccupations, en se centrant sur le type de site qui pouvait leur fournir les renseignements nécessaires. Un deuxième groupe a eu la même démarche. Ils ont également pris en compte les utilisateurs-cibles du site, choisissant un site à destination de débutants. Ce site leur apportait les informations sur le coût des installations, adaptées à leur niveau de compréhension.

\section{Synthèse globale et discussion}

Les résultats obtenus sont évidemment à moduler car ils ont été établis sur des données de justification de choix de documents, sélectionnés par des groupes de 3 à 4 élèves et sur une seule classe de cinquième. D'autres données ont été récoltées également sur les documents rejetés dans les groupes. Il serait pertinent, nous semble-t-il, de nous interroger sur les causes des désaccords et des consensus obtenus dans la sélection des documents, car cela a pu avoir un impact sur les critères de sélection. D’autres part, nous n'avons pas de données sur la position initiale des élèves quant au choix du Wi-Fi ou du filaire. Nous sommes donc consciente que cela a pu avoir également un impact sur la sélection des documents et sur les désaccords ou les consensus dans les groupes. Dans les deux cas, il nous semble que l'opinion initiale peut avoir influencé le choix des sources et donc le type d'informations retenues. C'est pourquoi la proposition faite par les élèves dans la recherche de Witzig et al. (2011) nous semble d'autant plus pertinente: éclaircir en premier lieu l'opinion initiale sur la question controversée afin d'apporter des éléments explicatifs supplémentaires.

En ayant ces limites à l'esprit, nous pouvons dire, à l'issue de cette étude, que ces 29 élèves ont été majoritairement dans une attitude évaluatrice. Cependant, ils sont restés centrés sur l'évaluation du contenu du document. L'évaluation de l'information en elle-même est très peu faite. Les auteurs des documents et, plus encore, le site source du document sont peu évalués.

Lorsqu'ils l'ont fait, les élèves ont attribué aux journalistes de santé une neutralité et une compétence à propos des informations délivrées. Leurs réponses ont été l'expression d'une absence de remise en cause de ces acteurs spécifiques. Cela met au jour que la majorité de ces jeunes élèves n'a pas de connaissances des intérêts et enjeux qui se jouent dans la sphère médiatique. Nous pouvons même dire que, dans le cas où ils ont évalué l'autorité journalistique, leur ignorance des 
intérêts associés à la diffusion d'informations a même contribué à renforcer leur confiance dans le contenu du document. Nous pouvons aussi nous questionner sur la compréhension effective du contenu, bien que ces élèves déclarent choisir un document parce qu'il est compréhensible.

Ce phénomène est aussi à noter lorsque les documents sont associés à des scientifiques. Même si quelques élèves évaluent la source, leur croyance en la neutralité des scientifiques et les intentions louables qu'ils leur attribuent ont contribué à renforcer la confiance qu'ils ont dans le contenu du document. La remise en cause des scientifiques, experts et organisations pour lesquels les élèves ne conçoivent pas d'intérêts personnels, économiques ou politiques est un point qui résiste. Les élèves persistent dans une vision «naïve» de ces autorités dont certaines, sous couvert de l'État, n'auraient que des intentions bienveillantes envers la population, notamment sur des questions de santé publique.

Même si peu d'élèves ont montré une attitude d'acceptation directe du document, leurs justifications ont porté sur l'impossibilité d'en trouver d'autres sur le domaine d'expertise traité.

Si nous confrontons ces résultats à ceux de Kolstø (2001), nous pouvons dire que l'expertise des documents extraits du web differe très peu d'une expertise de document "papier». Si les élèves ne se questionnent pas sur le site source, cela interroge en amont la nécessité de l'apprentissage de la recherche dans le web avant même de se pencher sur leur capacité d'expertise. Cela permettrait peut-être d'éviter que ce soit la première page et le premier site donnés par le moteur de recherche qui orientent le choix d'informations.

Très peu d'élèves sont dans une posture d'évaluation autonome (Kolstø, 2001). Confronter des informations contradictoires et des points de vue différents apparait pourtant comme fondamental dans l'expertise documentaire impliquant des sciences en construction.

Les critères d'évaluation du contenu des documents sélectionnés, utilisés par les jeunes élèves de notre étude, ont des similitudes avec ceux choisis par des étudiants de première année d'École d'ingénieur en agronomie (Witzig et al., 2011). Nous aurions pu penser que des élèves ingénieurs scientifiques auraient eu recours à des critères d'évaluation plus poussés que des élèves de 12 ans non spécialistes des sciences. Nos résultats convergent avec les leurs sur différents points. Witzig et al. (2011) ont établi que les étudiants se centrent peu sur l'information elle-même mais sur des critères tels que la facilité de compréhension, les détails, les données factuelles et la quantité d'informations. Les élèves de notre étude ont accordé également plus de crédit aux documents comportant des données factuelles et rejeté ceux qui étaient l'expression d'une opinion. Ils attribuent des compétences spécifiques aux journalistes de santé. Nos résultats comme ceux de Witzig et al. (2011) mettent en évidence une confiance attribuée aux sites médicaux, quel que soit l'âge des apprenants. Cependant, Kolstø (2001) et Witzig et al. (2011) rapportent que quelques élèves semblent sceptiques quant à l'objectivité des scientifiques. Aucun des élèves de notre étude ne l'a remise en 
question, qu'ils soient d'ailleurs dans une attitude d'acceptation ou d'évaluation de l'autorité. Cela peut s'expliquer par la jeunesse des élèves mais aussi par une survalorisation des sciences et des discours des experts que soulignent plusieurs recherches dans le champ des QSSs (Bader, 2003; Lewis, Leach \& Wood-Robinson, 1999; Molinatti, 2007; Molinatti, Girault \& Hammond, 2010). Si nous sommes d'accord sur le fait que cette confiance accordée aux scientifiques peut être attribuée à cette survalorisation des sciences, cela n'explique pas la confiance qu'ils accordent aux médias. Si caractériser les attitudes des élèves comme étant évaluatrices ou d'acceptation (Kolstø, 2001) donne à comprendre comment ils font, il nous semble primordial de poursuivre l'investigation sur le choix des critères d'évaluation retenus par les élèves.

L'expertise documentaire est ici basée sur des critères bâtis sur une connaissance rudimentaire des pratiques des auteurs des documents. Les connaissances des élèves sur les acteurs des controverses et sur les auteurs des informations sont à explorer. Ainsi, cela renvoie pour une part à la connaissance de la nature des sciences. D'autre part, cela interroge la connaissance des pratiques de communication et de diffusion des informations, des liens qu'entretiennent certains acteurs et certains médias avec les décisions gouvernementales, de leurs financements et des intérêts qui se jouent autour des QSSs.

Nous avons mis en avant la difficulté des élèves à expertiser des documents liés principalement à la méconnaissance des intérêts et des pratiques des acteurs engagés dans la controverse ainsi que des auteurs de documents - qui peuvent être d'ailleurs également des acteurs de la controverse. C'est d'ailleurs une des difficultés majeures quand on traite de QSS et de sciences en train de se faire. En effet, Latour (2007) montre comment tout groupe qui élabore des connaissances a des intérêts, des valeurs, des visions du monde qui ont un impact sur l'interprétation des données. Les décisions sont alors prises également en fonction de raisons politiques.

Cela nous conduit à questionner les savoirs à prendre en compte dans la scolarisation des QSSs au-delà des "savoirs naturels et sociaux", des "savoirs et pratiques dans des groupes» et des «savoirs scolaires» développés par Albe (2007). Il paraît nécessaire, à l'issue de cette étude, de prendre en compte des savoirs sur les différents acteurs engagés dans les QSSs et sur les auteurs des ressources documentaires utilisées en classe. Leurs rôles, leurs financements, leurs intérêts, les modes de diffusion et de communication qu'ils emploient et les liens qu'ils entretiennent avec les décisions de politiques gouvernementales sont à développer pour que les élèves puissent avoir en main tous les éléments d'une expertise documentaire informée. Même si des choix curriculaires s'imposent en dehors de ces considérations, l'École peut-elle taire ces aspects de la construction des savoirs? Cela reviendrait alors à renoncer à une $\mathrm{EC}$ critique et à maintenir des élèves, mêmes adultes et spécialistes des sciences dans une expertise de l'information comparable à celles de jeunes élèves non spécialistes. Ces savoirs sur les auteurs de documents et sur les acteurs engagés dans la QSS traitée pourraient 
ainsi peut-être permettre à des élèves d'appréhender les enjeux socio-politiques, éthiques et scientifiques indissociables des innovations scientifiques et techniques. Consécutivement, cela pourrait avoir un impact sur les prises de décision de n'importe quel citoyen, si elles sont étayées par une expertise plus perspicace de l'information.

Il est nécessaire de considérer une éducation aux médias et à l'expertise de documents qui prenne en compte ces savoirs. L'enjeu est d'apporter les outils nécessaires aux élèves pour qu'ils puissent appréhender les pratiques, les intérêts et les enjeux de la diffusion de l'information dans le but de se construire une citoyenneté avertie.

\section{Note}

1 Voir par exemple: http://controverses.sciences-po.fr/archive/hypersensibiliteondes/index. html (consulté le 3 novembre 2016)

\section{Ré férences}

Albe, V. (2007). Des controverses scientifiques socialement vives en éducation aux sciences. État des recherches et Perspectives. Mémoire de synthèse pour l'Habilitation à diriger des Recherches, Université Lyon 2, Lyon.

Audigier, F. (1999). L'éducation civique dans l'enseignement secondaire: quelques repères Historiques. L'école du citoyen. Iregh, 7, 11-25.

Audigier, F. (2000). Instruction civique, éducation civique, éducation à la citoyenneté, Education aux citoyennetés... Changement de nom, changement de contenu? In L. Pfander-Mény \& J.-G. Lebeau (Éd.), Vers une citoyenneté européenne. Dijon: CRDP, 23-40.

Bader, B. (2003). Interprétation d'une controverse scientifique: stratégies argumentatives d'adolescentes et d'adolescents québécois. Revue canadienne de l'enseignement des sciences, des mathématiques et des technologies, 3, 231-250.

Barrué, C. \& Albe, V. (2013). Citizenship education and socioscientific issues: Implicit concept of Citizenship in the Curriculum, views of French Middle School Teachers, Science \& Education, 22, (5), 1089-1114.

Barrué, C. (2014). L'enseignement des thèmes de convergence au collège: mise en débat d'une question socioscientifique en classe pour une Education Citoyenne. Thèse de Doctorat ENS de Cachan Université Paris Saclay, École Doctorale de Sciences Patiques, Cachan.

Barthe, Y. (2006). Le pouvoir d'indécision. La mise en politique des déchets nucléaires. Paris: Economica, coll. Etudes politiques.

Bonneuil, C. (2004). Les transformations des rapports entre sciences et société en France depuis la seconde guerre mondiale: un essai de synthèse. Article présenté au Colloque Sciences Médias et Société, Lyon ENS-LSH, 15-16 Juin 2004.

Böttcher, F. \& Meisert, A. (2013). Effects of Direct and Indirect Instruction on Fostering Decision-Making Competence in Socioscientific Issues, Research of Science Education, 43, 479-506.

Bourdieu, P. (1996). Sur la télévision. Paris: Raisons d'agir.

Byrne, J., Ideland. M., Malmberg, M. \& Grace, M. (2014). Climate Change and Everyday Life: Repertoires children use to negotiate a socio-scientific issue. International Journal of Science Education, 36, (9), 1491-1509.

Callon, M., Lascoumes, P. \& Barthe, Y. (2001). Agir Dans Un Monde Incertain: Essai Sur La Démocratie Technique. Paris: Seuil.

Dewey, J. (2003). Le Public Et Ses Problèmes. Pau: Farrago. 
Grace, M. (2009). Developing High Quality Decision-Making Discussions About Biological Conservation in a Normal Classroom Setting. International Journal of Science Education, 31, 551-570.

Halimi, S. (1999). Ces débats médiatiquement corrects. Consulté le 23 avril 2009 sur le site du Monde diplomatique: http://www.monde-diplomatique.fr/1999/03/HALIMI/11798

Hingant, B. (2013). Les nanotechnologies dans l'enseignement secondaire: Une recherche sur la compréhension des controverses "nanos» par des lycéens. Thèse de Doctorat, ENS de Cachan, Institut Néel de Grenoble, École Doctorale de l'Ingénierie de la Santé, de la Cognition et de l'Environnement de Grenoble.

Hodson, D. (2003). Time for action: Science education for an alternative future. International Journal of Science Education, 25, 645- 670.

Hodson, D. (2010). Science Education as a Call to Action. Canadian Journal of Science, Mathematics and Technology Education, 10, 197- 206.

Hogan, K. (2002). Small groups' ecological reasoning while making an environmental management decision. Journal of Research in Science Teaching, 39, 341-368.

Jiménez-aleixandre, M.-P. \& Pereiro-Muñoz, C. (2002). Knowledge producers or knowledge consumers? Argumentation and decision making about environmental management. International Journal of Science Education, 24, 1171-1190.

Kelly, T. (1986). Discussing controversial issues: four perspectives on the teacher's role. Theory and Research in Social Education, 14, 113-138.

Klosterman, M.L., \& Sadler, T. D. (2008). Information literacy for science education: Evaluating web-based materials for socioscientific issues. Science Scope, 31, (7), 18-21.

Klosterman, M.L. \& Sadler, T. D. (2010). Multi-level Assessment of Scientific Content Knowledge Gains Associated with Socioscientific Issues-based Instruction. International Journal of Science Education, 32, (8), 1017-1043.

Kolstø, S. D. (2000). Consensus projects: Teaching science for citizenship. Research Report. International Journal of Science Education, 22, 645-664.

Kolstø, S. D. (2001). Scientific literacy for citizenship: Tools for dealing with the science dimension of controversial socioscientific issues. Science Education, 85, 291-310.

Latour, B. (2007). Cours de description des controverses. Consultable sur le site de l'École des mines de Paris. Consulté le 6 novembre 2016 dans http://controverses.ensmp.fr

Levinson, R. (2004). Teaching Bioethics in Science: Crossing a bridge too Far? Canadian Journal of Science, Mathematics and Technology Education, 4, 353-369.

Lewis, J., Leach, J. \& Wood-Robinson, C. (1999). Attitude des jeunes face à la technologie génique. In L. Simonneaux (Éd.), Les biotechnologies à l'école. Dijon: Educagri éditions.

Molinatti, G. (2007). Médiation des sciences du cerveau: Approche didactique et communicationnelle de rencontres entre neuroscientifiques et lycéens. Thèse de Doctorat, Muséum National d'Histoire naturelle de Paris.

Molinatti, G., Girault, Y. \& Hammond, C. (2010). High School Students Debate the Use of Embryonic Stem Cells: The influence of context on decision-making. InternationalJournal of Science Education, 32, (16), 2235-2251.

Pedretti, E. (1999). Decision making and STS education: exploring scientific knowledge and social responsibility in schools and science centers through an issues-based approach. Journal of School Science and Mathematics, 99, 174-181.

Sadler, T. D. (2004). Informal reasoning regarding socioscientific issues: A critical review of research. Journal of Research in Science Teaching, 41, 513-536.

Sadler, T. D. (2009). Situated learning in science education: socio-scientific issues as contexts for practice. Studies in Science Education, 45, (1), 1- 42.

Simonneaux, L., Ducamp, Ch., Albe, V., Simonneaux, J. \& Hirtzlin, N. (2005). La perception des sciences par des lycéens est-elle modifiée par la présentation par des chercheurs de leurs travaux? Paper presented at the biannual meeting of Association pour la recherche en didactique des sciences et des techniques, Lyon. 
Witzig, S.B., Halverson, K.L., Siegel, M.A. \& Freyermuth, S.K. (2011). The Interface of Opinion, Understanding and Evaluation While Learning About a Socioscientific Issue. International Journal of Science Education, 35, (15).

Mots-clés: Questions socioscientifiques, éducation citoyenne, expertise de l'information

\section{Diskussion über die Behandlung sozialwissenschaftlicher Fragestellungen: Untersuchung der Information im Rahmen einer staatsbürgerlichen Bildung}

\section{Zusammenfassung}

Jede Überlegung zur Bürgerbeteiligung über Entscheidungen, die umstrittene wissenschaftliche Fragestellungen betreffen, sollte zu der Frage führen, ob man diese Themen auch in der Schule behandeln soll. Schülerinnen und Schüler im Alter von 11 bis 12 Jahren nehmen daher an einer staatsbürgerlichen Konferenz (speziell für Schülerinnen und Schüler) teil. Wenn man nach kritischer staatsbürgerlicher Bildung strebt, ist es wichtig, sich für die dokumentarische Analyse zu interessieren. Was sind die Kriterien der Schülerinnen und Schüler, wenn sie Dokumente und Informationsquellen analysieren? Zwar können die Lernenden die Dokumentinhalte untersuchen, aber nur wenige können sie auch bewerten und in den Gesamtkontext einordnen. Im Unterricht sollte die Informationsanalyse eingeführt werden, damit die Schülerinnen und Schüler die Komplexität und das Zusammenspiel sozialer und wissenschaftlicher Kontroversen verstehen können.

Schlagworte: Wissenschaftliche Kontroversen, Bürgerschaftserziehung, Informationsforschung

\section{Dibattito su una questione socio-scientifica: le competenze informazionali nell'ambito di un'Educazione Civica}

\section{Riassunto}

Le riflessioni sul contributo dei cittadini alle decisioni relative a questioni scientifiche controverse devono mettere in causa la scolarizzazione di quest'ultime. Questo studio si basa sull'impegno di alunni tra gli undici e i dodici anni durante un'assemblea civica adattata al contesto scolastico. Per raggiungere l'obiettivo di un'Educazione Civica critica, sembra fondamentale indagare sulle competenze di gestione dei documenti e delle informazioni (information literacy). Quali sono i criteri utilizzati dagli alunni nella valutazione dei documenti e delle fonti 
d'informazione quando le conoscenze scientifiche sono controverse? Se gli alunni valutano strategicamente il contenuto delle questioni nei documenti, solo pochi dedicano un'attenzione accurata alle fonti. Quindi, bisogna dedicare un'attenzione approfondita alla valutazione dell'informazione affinché gli alunni possano capire la complessità delle sfide legate alle controverse sociali e scientifiche.

Parole chiavi: Questioni socio-scientifiche, Educazione Civica, information literacy

\section{Debate on a socioscientific issue: Evaluation of information in a framework of Citizenship Education}

\section{Abstract}

If citizen participation in decision-making about socioscientific issues is considered as legitimate, the schooling of it should be question. In this research, 11-12 years old students were engaged in a citizens' conference adapted to the school context. In an aim of Citizenship Education, it is crucial to focus on the documentary expertise. What are the criteria used by students to evaluate documents and information sources? If students were in an evaluative strategy for the content of documents, a few of them evaluated the sources. Information literacy also should be considered in order to enable students to understand the complexity of such issues because of the social and scientific aspects of socioscientific issues.

Keywords: Socioscientific issues, Citizenship, Education, Information literacy 
